\title{
Observation of Entropic Effect on Conformation Changes of Complex Systems Under Well-Controlled Temperature Condition
}

\author{
Xue-Bin Wang, Jie Yang, and Lai-Sheng Wang* \\ Department of Physics, Washington State University, 2710 University Drive, Richland, \\ WA 99354 and Chemical \& Materials Sciences Division, Pacific Northwest National \\ Laboratory, MS K8-88, P. O. Box 999, Richland, WA 99352
}

Supporting information 
Figure S1. Deconvolution of the temperature dependent photoelectron spectra of ${ }^{-} \mathrm{O}_{2} \mathrm{C}_{(}\left(\mathrm{CH}_{2}\right)_{6} \mathrm{CO}_{2}^{-}$ $\left(\mathrm{H}_{2} \mathrm{O}\right)_{14}$. The red curve is the spectrum at $18 \mathrm{~K}$, representing the linear conformation. The blue curve is the difference spectrum between the total spectrum (black) at each temperature and that of the $18 \mathrm{~K}$ spectrum, representing the spectrum of the folded conformation.
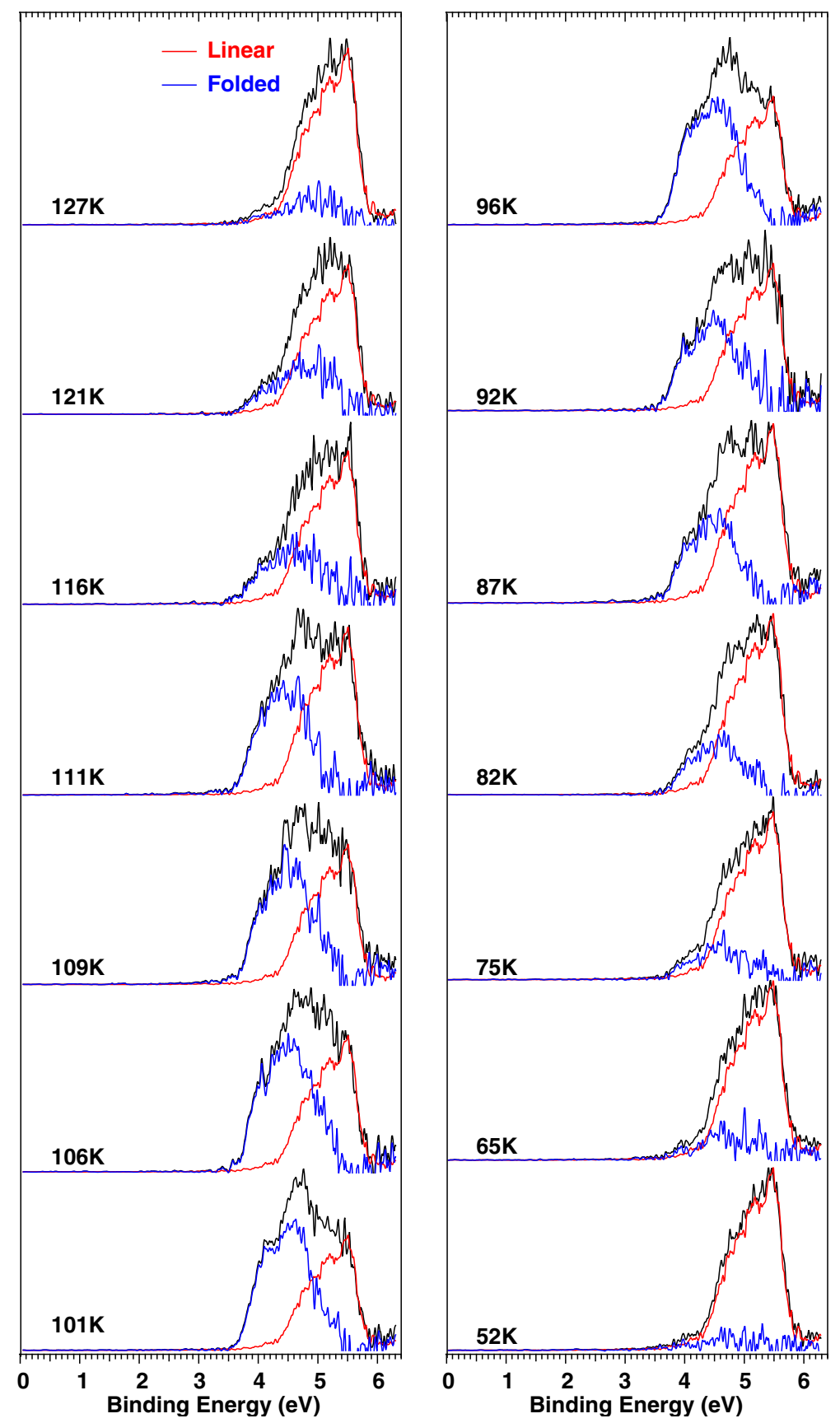